Physics Vol. 3, No. 4, pp. 165-180, 1967. Physics Publishing Co. Printed in Great Britain.

\title{
GENERALIZED ALgEbra OF CURRENTS AND REgGe POLES*
}

\author{
SUSUMU OKUBO \\ Department of Physics and Astronomy, University of \\ Rochester, Rochester, New York \\ (Received 25 January 1967 )
}

\begin{abstract}
Ry using the generalized algebra of currents based upon commutators on the light cone, one can show that one can re-interpret Regge trajectories in terms of our generalized commutators. In this way, the correspondence between our theory and Regge's is one to one, and we can derive many results of Regge poles without invoking the concept of complex angular momentum. Besides, many sum rules for the high energy scattering cross-sections are obtained.
\end{abstract}

\section{Introduction}

AT THE moment, there are several successful approaches to problems involving strong interactions. These are the dispersion theory, Regge poles and the algebra of currents. Of all these three approaches, the dispersion theory is the most universal in its application and hence extensively utilized even for problems involving Regge poles and the algebra of currents. Apart from this use of the dispersion theory, it looked until now that Regge poles and the algebra of currents have no relation of any kind whatever. Indeed, this was not surprising in view of the fact that the former is essentially connected with high energy phenomena while the latter is so far restricted to giving low-energy theorems. However, this is not really the whole story. In a previous paper [1], it has been shown that an algebra of currents among spatial components of electromagnetic currents may give rise to a high energy theorem. Unfortunately, this technique is not easily generalizable for more interesting situations.

In this note, we shall present another approach where we generalize the concept of the algebra of currents not only on the equal-time but also on more general commutators on the light cone. We shall see that in this way, we are naturally led into investigating the so-called $t$ channel and that we may obtain many results of the Regge pole model. For instance, the BargerRubin relation [2] for high energy meson-baryon scattering cross-sections is easily obtainable, together with many others. In fact, it is possible in our theory to re-interpret the Regge trajectories in terms of generalized commutators on the light cone. Although some assumptions must be made for such an interpretation, the whole situation appears to be quite promising and it is hoped that further investigations may clear up these points.

- This work is supported in part by the Atomic Energy Commission. 


\section{Generalized Conmutators and Sum Rules}

We shall consider scattering of an octet of pseudoscalar mesons by some targets which we need not specify at the moment. Now, using the tensor notation [3] of representations of the SU(3) group, one may denote the meson field by a traceless tensor $\varphi_{b}^{a}(x),(a, b=1,2,3)$. Then, the standard technique enables us to write down the scattering amplitude $T(i \rightarrow f)$ where an incoming meson $\varphi_{a}^{b}$ with 4-momentum $k$ is scattered by a target specified by $|\alpha\rangle$ and an out,going meson $\varphi_{d}^{c}$ with 4-momentum $k^{\prime}$ emerges together with a recoiled residual taget $|\beta\rangle$.

$$
\begin{aligned}
& T(i \rightarrow f)=i\left(4 k_{0} k_{0}^{\prime}\right)^{-1 / 2} \int d^{4} x \exp \left[i \frac{k+k^{\prime}}{2} x\right] \times \\
& \quad \times\left\{\left\langle\beta\left|\theta\left(-x_{0}\right)\left[j_{b}^{a}\left(\frac{x}{2}\right), j_{d}^{c}\left(-\frac{x}{2}\right)\right]\right| \beta>-\left\langle\beta\left|R_{b d}^{a c}(x)\right| \alpha>\right\}\right.\right.
\end{aligned}
$$

where we defined the pseudoscalar current $j_{b}^{a}(x)$ by

$$
j_{b}^{a}(x)=\left(\mu^{2}-\square \varphi_{b}^{a}(x)\right.
$$

and $R_{b d}^{a c}(x)$ is a sum of equal-time commutators given by

$$
\begin{aligned}
R_{b d}^{a c}(x) & =2 \delta\left(x_{0}\right)\left[j_{b}^{a}\left(\frac{x}{2}\right), \frac{\partial}{\partial x_{0}} \phi_{d}^{c}\left(-\frac{x}{2}\right)\right]+2 \delta\left(x_{0}\right)\left[\frac{\partial}{\partial x_{0}} \Phi_{b}^{a}\left(\frac{x}{2}\right), j_{d}^{c}\left(-\frac{x}{2}\right)\right]+ \\
& +\frac{1}{2} \frac{\partial \delta\left(x_{0}\right)}{\partial x_{0}}\left[j_{b}^{a}\left(\frac{x}{2}\right), \phi_{d}^{c}\left(-\frac{x}{2}\right)\right]+\frac{1}{2} \frac{\partial \delta\left(x_{0}\right)}{\partial x_{0}}\left[\Phi_{b}^{a}\left(\frac{x}{2}\right), j_{d}^{c}\left(-\frac{x}{2}\right)\right]
\end{aligned}
$$

Actually, the meson mass $\mu$ in equation (2) need not be the same for all mesons and may be replaced by $\mu_{b}^{a}$.

Now, it is easy to show that the $R_{b d}^{a c}(x)$ term does not contribute at all to the imaginary part of the forward scattering amplitude, and hence to the total scattering cross section, by the optical theorem. Thus, it is conceivable that its effects may be negligible in the high energy limit. Indeed, we shall justify this conjecture in the next section. However, we need not assume this in this section.

Let us consider the first term on the right hand side (hereafter referred to as R.H.S.) of equation (1). For the high energy limit, we let $k_{0} \approx k_{0}^{\prime} \rightarrow \infty$ with a fixed momentum transfer. Then, the main contribution to the scattering amplitude would come [4] from the most singular parts of the retarded commutator since non-singular parts are expected to give vanishingly smal contributions in that limit thanks to the Rieman-Lebesgue lemma on the Fourier transform. Ordinarily, we believe that these singular parts are concentrated on the light cone $x^{2}=0$ because of the micro-causality. Then, we shall show in the next section that indeed one may obtain a 
iffraction shrinkage from such assumptions. Further, we assume that on the light cone $x^{2}=0$, he commutator will contain only unitary singlet and octet components, i.e. it is assumed that t $x^{2}=0$, we have

$$
\left[j_{b}^{a}\left(\frac{x}{2}\right), j_{d}^{c}\left(-\frac{x}{2}\right)\right]=S_{b d}^{a c}(x)+F_{b d}^{a c}(x)+D_{b d}^{a c}(x)
$$

here

$$
\begin{gathered}
S_{b d}^{a c}(x)=\left[\delta_{d}^{a} \delta_{b}^{c}-\frac{1}{3} \delta_{b}^{a} \delta_{d}^{c}\right] Q_{0}(x), \\
F_{b d}^{a c}(x)=\delta_{d}^{a} Q_{b}^{c}(x)-\delta_{b}^{c} Q_{d}^{a}(x), \\
D_{b d}^{a c}(x)=\delta_{d}^{a} \bar{Q}_{b}^{c}(x)+\delta_{b}^{c} \bar{Q}_{d}^{a}(x)-\frac{2}{3} \delta_{b}^{a} \bar{Q}_{\frac{c}{d}}^{c}(x)- \\
-\frac{2}{3} \delta_{d}^{c} \bar{Q}_{b}^{a}(x)+\frac{2}{9} \delta_{b}^{a} \delta_{d}^{c} \bar{Q}_{f}^{f}(x) .
\end{gathered}
$$

Note that $S_{b d}^{a c}(x), F_{b d}^{a c}(x)$ and $D_{b d}^{a c}(x)$ correspond to the unitary singlet, an $f$-type octet, and a d-type octet, respectively. In general, we should have three more terms on the R.H.S. of equation (4), corresponding to $10, \overline{10}$ and 27 dimensional representations of the SU(3) group. It is morth emphasizing that we did not take $Q_{b}^{a}(x)$ and $\bar{Q}_{b}^{a}(x)$ in equation (5) to be traceless for a reason to be explained later, although we can always normalize them to be traceless by suitable redefinitions.

In this section, we do not assume any explicit form for $Q_{0}(x), Q_{b}^{a}(x)$ and $\bar{Q}_{b}^{a}(x)$. Now, we shall present a motivation for our assumption equation (4). We know that for an equal-time commutator, we shall have an analogue of equation (4) for a commutator of two octet currents. For instance, equal-time commutation relations among spatial components of vector or axial-vector octet currents in the ordinary algebra of currents [5] have the form of equation (4).

As we shall see shortly, this assumption appears to be somehow intimately related to the Regge pole model where contributions from $Q_{0}(x), Q_{b}^{a}(x)$ and $\bar{Q}_{b}^{a}(x)$ may correspond to exchanges of the Pomeranchukon, the $1^{-}$vector nonet and the $2^{+}$tensor nonet trajectories, respectively. We may also remark that the importance of the $t$-channel in the high energy phenomena is naturally incorporated in our theory.

So far, we have used the terminology of the SU(3) group, thus implicitly assuming the validity of the SU(3) symmetry. However, in reality we need not assume its exact validity. Indeed, one may assume equation (4) independently of the SU(3) symmetry at $x^{2}=0$. Especially, it may hold valid even if the SU(3) is badly broken. This situation is again quite analogous to the ordinary algebra of currents [5]. In fact, as we shall see soon, this may explain why the Barger-Rubin relation [2] and others are well satisfied experimentally in spite of possible large SU(3) breaking. In view of this, we shall not assume the exact validity of the SU(3) 
symmetry in this paper unless otherwise so stated.

When we have to take account of the contribution from the $R_{b d}^{a c}(x)$ term of equation (3), then we once again assume that $R_{b d}^{a c}(x)$ is a sum of unitary singlet and octets as in equation (4). In this case, this assumption is more plausible since we are considering equal-time commutators. However, for simplicity, we shall omit to mention the contribution from this term since most conclusions presented in this section are really unaffected by its inclusion. Only when its in. clusion may change our results shall we refer to it, although its effects are actually negligible as we shall show in the next section.

Now, it is easy to calculate the scattering amplitude in the high energy limit from equation (1). (4) and (5). For instance, one may get

$$
\begin{aligned}
& T\left(\pi^{ \pm}{ }_{\alpha} \pi^{ \pm} \beta\right)=\left\langle\beta\left|Q_{0}\right| \alpha\right\rangle \pm\left\langle\beta\left|\left(Q_{2}^{2}-Q_{1}^{1}\right)\right| \alpha\right\rangle+\left\langle\beta\left|\left(\bar{Q}_{1}^{1}+\bar{Q}_{2}^{2}\right)\right| \alpha\right\rangle \\
& T\left(k^{ \pm} \alpha \rightarrow k^{ \pm} \beta\right)=\left\langle\beta\left|Q_{0}\right| \alpha\right\rangle \pm\left\langle\beta\left|\left(Q_{3}^{3}-Q_{1}^{1}\right)\right| \alpha\right\rangle+\left\langle\beta\left|\left(\bar{Q}_{1}^{1}+\bar{Q}_{3}^{3}\right)\right| \alpha\right\rangle \\
& T\left(k^{0} \alpha \rightarrow k^{0} \beta\right)=\left\langle\beta\left|Q_{0}\right| \alpha\right\rangle+\left\langle\beta\left|\left(Q_{2}^{3}-Q_{2}^{2}\right)\right| \alpha\right\rangle+\left\langle\beta\left|\left(\bar{Q}_{2}^{2}+\bar{Q}_{3}^{3}\right)\right| \alpha\right\rangle \\
& T\left(\bar{k}^{0} \alpha \rightarrow \bar{k}^{0} \beta\right)=\left\langle\beta\left|Q_{0}\right| \alpha\right\rangle-\left\langle\beta\left|\left(Q_{3}^{3}-Q_{2}^{2}\right)\right| \alpha\right\rangle+\left\langle\beta\left|\left(\bar{Q}_{2}^{2}+\bar{Q}_{3}^{3}\right)\right| \alpha\right\rangle
\end{aligned}
$$

where for simplicity we omitted the integration symbol together. with other multiplicative factors. From equation (6), one immediately finds the following:

$$
\begin{aligned}
T\left(\pi^{-} \alpha \rightarrow \pi^{-} \beta\right)-T\left(\pi^{+} \alpha \rightarrow \pi^{+} \beta\right) & =T\left(k^{-} \alpha \rightarrow k^{-} \beta\right)-T\left(k^{+} \alpha \rightarrow k^{+} \beta\right)+ \\
& +T\left(k^{0} \alpha \rightarrow k^{0} \beta\right)-T\left(\bar{k}^{0} \alpha \rightarrow \bar{k}^{0} \beta\right) .
\end{aligned}
$$

Restricting ourselves to the discussion of the forward elastic scattering $(\alpha=\beta)$ and taking the imaginary parts of both sides, one finds the following relation among total scattering cross-sections because of the optical theorem:

$$
\sigma_{t}\left(\pi^{-} \alpha\right)-\sigma_{t}\left(\pi^{+} \alpha\right)=\sigma_{t}\left(k^{-} \alpha\right)-\sigma_{t}\left(k^{+} \alpha\right)+\sigma_{t}\left(k^{0} \alpha\right)-\sigma_{t}\left(\bar{k}^{0} \alpha\right) .
$$

Further, as a special case of equation (8), one gets the familiar Barger-Rubin relation [2]

$$
\sigma_{t}\left(\pi^{-} p\right)-\sigma_{t}\left(\pi^{+} p\right)=\sigma_{t}\left(k^{-} p\right)-\sigma_{t}\left(k^{+} p\right)+\sigma_{t}\left(k^{+} n\right)-\sigma_{t}\left(k^{-} n\right)
$$

where we used the charge symmetry for the baryon-pion system. Note that in the ordinary derivation of this equation, we assume the validity of the exact su(3) symmetry together with exchanges of Reggeized unitary singlet and octets particles in the $t$-channel. However, in our derivation, the exact validity of the $\mathrm{SU}(3)$ is not necessarily assumed, although the validity of equation (9) is maintained only in the high energy limit where one can neglect the mass differences of incoming pion and kaons in comparison with the incident energy. This may account 
for the satisfactory agreement of equation (9) with experiment [2] in spite of possible large violations of the $s U(3)$ symmetry.

One may remark also that equation (8) must be valid for any state $|\alpha\rangle$. Hence, one mas replace the proton and the neutron in equation (9) by $\mathrm{He}^{3}$ and $\mathrm{H}^{3}$ nuclei, respectively. This is rather an interesting situation since ordinarily one expects a complication due to possible occurrence of Regge cuts [6] for complex nuclear scattering. In spite of it, our theory predicts that equation (8) for complex nuclei must hold valid.

We can derive many other relations for differential cross-sections also. As an example, let us calculate

$$
\begin{aligned}
& T\left(\pi^{-} \alpha \rightarrow \pi^{0} \beta\right)=-\sqrt{2}\left\langle\beta\left|Q_{1}^{2}\right|_{\alpha}\right\rangle \\
& T\left(\pi^{-} \alpha \rightarrow \eta \beta\right)=\sqrt{\frac{2}{3}}\left\langle\beta\left|\bar{Q}_{1}^{2}\right|_{\alpha}\right. \\
& T\left(\dot{k}^{0} \alpha \rightarrow k^{+} \beta\right)=-\left\langle\beta\left|Q_{1}^{2}\right| \alpha\right\rangle+\left\langle\beta\left|\bar{Q}_{1}^{2}\right|_{\alpha}\right\rangle \\
& T\left(k^{-} \alpha \rightarrow \bar{k}^{0} \beta\right)=+\left\langle\beta\left|Q_{1}^{2}\right| \alpha\right\rangle+\left\langle\beta\left|\bar{Q}_{1}^{2}\right|_{\alpha}\right\rangle
\end{aligned}
$$

in the same notation as before. Then, one derives two relations:

$$
\begin{aligned}
& -\sqrt{2} T\left(\pi^{-} \alpha \rightarrow \pi^{0} \beta\right)=T\left(k^{-} \alpha \rightarrow \bar{k}^{0} \beta\right)-T\left(k^{0} \alpha \rightarrow k^{+} \beta\right) . \\
& -\sqrt{6} T\left(\pi^{-} \alpha \rightarrow \eta \beta\right)=T\left(k^{-} \alpha \rightarrow \bar{k}^{0} \beta\right)+T\left(k^{0} \alpha \rightarrow k^{+} \beta\right) .
\end{aligned}
$$

from which one obtains the following relation among differential scattering cross-sections:

$$
\begin{aligned}
& \frac{d}{d Q} \sigma\left(k^{-} \alpha \rightarrow \bar{k}^{0} \beta\right)+\frac{d}{d Q} \sigma\left(k^{0} \alpha \rightarrow k^{+} \beta\right)= \\
& \left.=\frac{d}{d Q} \sigma\left(\pi^{-} \alpha \rightarrow \pi^{0} \beta\right)+3 ; \quad-\alpha \rightarrow \eta \beta\right) .
\end{aligned}
$$

Similarly, one gets another sum rule:

$$
\begin{aligned}
& \frac{d}{d \Omega} \sigma\left(k^{+} \alpha \rightarrow k^{0} \beta\right)+\frac{d}{d \Omega} \sigma\left(\bar{k}^{0} \alpha \rightarrow k^{-} \beta\right)= \\
& \quad=\frac{d}{d \Omega} \sigma\left(\pi^{+} \alpha \rightarrow \pi^{0} \beta\right)+3 \frac{d}{d \Omega} \sigma\left(\pi^{+} \alpha \rightarrow \eta \beta\right) .
\end{aligned}
$$


It is easy to see that in the Regge pole model, the contributions from $Q_{0}(x), Q_{b}^{a}(x)$ and $\bar{Q}_{b}^{a}(x)$ correspond to exchanges of Pomeranchukon, $1^{-}$vector nonet and $2^{+}$tensor nonet trajectories. Actually, this correspondence is one to one as we see from equation (4) or from more direct calculations like equations $(10)$ and $(6)$. We shall come back to this point in more detail in the next section. Now, experimentally, the slopes of the Regge trajectories $\alpha(t)$ for both $1^{-}$ and $2^{+}$nonets are almost identical for particles with the same charge and strangeness. Tnen, due to the difference of the signature factors of the two trajectories, the contribution from $Q_{b}^{a}(x)$ must have a phase difference of $90^{\circ}$ from that of the $\bar{Q}_{b}^{a}(x)$ term. This implies that boti contributions add incoherently for differential cross-sections. For example, we should get then

$$
\frac{d}{d \Omega} \sigma\left(k^{-} \alpha \rightarrow \bar{k}^{0} \beta\right)=\frac{d}{d \Omega} \sigma\left(k^{0} \alpha \rightarrow k^{+} \beta\right)
$$

as one may see from equation (10). In the next section we shall show that this phase difference of $90^{\circ}$ is really related to the time-reversal invariance of the theory in our approach. We may also remark that this relation equation (14) and most of the similar relations obtained below are based not only upon this special assumption made in the above but also upon the fact that one may neglect the contribution from the $R_{b d}^{a c}$ term of equation (3). Note that the validity of equations (8), (9), (12) and (13) are independent of these assumptions.

Returning to the original discussion, equations (14) and (12) lead to

$$
2 \frac{d}{d \Omega} \sigma\left(k^{-} \alpha \rightarrow \bar{k}^{0} \beta\right)=\frac{d}{d \Omega} \sigma\left(\pi^{-} \alpha \rightarrow \pi^{0} \beta\right)+3 \frac{d}{d \Omega} \sigma\left(\pi^{-} \alpha \rightarrow \eta \beta\right) .
$$

Again, if we identify $|\alpha\rangle$ and $|\beta\rangle$ to be the proton and the neutron, respectively, one finds

$$
2 \frac{d}{d \Omega} \sigma\left(k^{-} p \rightarrow \bar{k}^{0} n\right)=\frac{d}{d \Omega} \sigma\left(\pi^{-} p \rightarrow \pi^{0} n\right)+3 \frac{d}{d \Omega} \sigma\left(\pi^{-} p \rightarrow \eta n\right)
$$

which together with equation (14) for $|\alpha\rangle=|p\rangle$ and $|\beta\rangle=|n\rangle$ is the relation obtained previously by Ahmadzadeh and Chan [7] under somewhat more stringent assumptions. They have also shown that equation (16) is well satisfied experimentally. Again, this may be understood better by our method since we need not assume the exact validity of the SU(3) symmetry. It is interesting to test equations (14) and (15) for the case where $|\alpha\rangle$ and $|\beta\rangle$ correspond to $\mathrm{He}^{3}$ and $\mathrm{H}^{3}$ nuclei, respectively.

In this connection, it may be worthwhile to make some observations on the validity of equation (14). Under the same assumptions, one can generalize it and easily show that the differential scattering cross-section for the reaction $b+\alpha \rightarrow b^{\prime}+\beta$ is the same as its crossingsymmetric reaction $\bar{b}^{\prime}+\alpha \rightarrow \bar{b}+\beta$ at high energy, provided that $b \neq b^{\prime}$ where $b$ and $b^{\prime}$ are any two members of the pseudoscalar meson octet and $\bar{b}$ and $\bar{b}^{\prime}$ are their antiparticles respectively. Thus, one would have

$$
\frac{d}{d \Omega} \sigma\left(b \alpha \rightarrow b^{\prime} \beta\right)=\frac{d}{d \Omega} \sigma\left(\bar{b}^{\prime} \alpha \rightarrow \bar{b} \beta\right), \quad\left(b \neq b^{\prime}\right)
$$

which contains equation (14) as a special case. We may call this principle the crossing-independent relation. Especially, one finds the following: 


$$
\begin{aligned}
& \frac{d}{d \Omega} \sigma\left(\pi^{+} \alpha \rightarrow k^{+} \beta\right)=\frac{d}{d \Omega} \sigma\left(k^{-} \alpha \rightarrow \pi^{-} \beta\right), \\
& \frac{d}{d \Omega} \sigma\left(\pi^{-} \alpha \rightarrow k^{0} \beta\right)=2 \frac{d}{d \Omega} \sigma\left(k^{-} \alpha \rightarrow \pi^{0} \beta\right)
\end{aligned}
$$

where for the derivation of the second of equation (18), we utilized equation (17) as well as the relation

$$
\frac{d}{d \Omega} \sigma\left(\bar{k}^{0} \alpha \rightarrow \pi^{+} \beta\right)=2 \frac{d}{d \Omega} \sigma\left(k^{-} \alpha \rightarrow \pi^{v} \beta\right)
$$

which can be obtained on the basis of equations (4) and (5).

We may notice that it is possible to generalize our crossing independent relation equation (17) for baryon-baryon scattering problems under similar assumptions to obtain

$$
\frac{d}{d \Omega} \alpha(p n \rightarrow n p)=\frac{d}{d \Omega} \sigma(p \bar{p} \rightarrow n \bar{n})
$$

which has already been noted by Ahmadzaden and Cnan [7] in the Regge pole model. However, this relation should not be good since $1^{+}$and $2^{-}$trajectories would contribute now to these reactions.

Finally, we observe that our ansatz equation (4) implies forbiddenness of all reactions involving changes of two units of electric or hypercharge; i.e.

$$
\frac{d}{d \Omega} \sigma\left(b \alpha \rightarrow b^{\prime} \beta\right)=0
$$

where we have either $|\Delta Q|=2$, or $|\Delta S|=2$ or $\Delta Q=-\Delta S= \pm 1$ and where

$$
\Delta Q=Q\left(b^{\prime}\right)-Q(b), \quad \Delta S=S\left(b^{\prime}\right)-S(b)
$$

are changes of electric charge and strangeness of the mesons involved. This validity of equation (20) is independent of the previous assumption of the experimental proximity of the Regge slopes of the $1^{-}$and $2^{+}$nonet trajectories. Also, it holds true even if we include the effect of the $R_{b d}^{a c}(x)$ term, provided that it consists again of unitary singlet and octets.

As a special application of equation (20), one finds

$$
\begin{aligned}
& \frac{d}{d \Omega} \sigma\left(k^{-} p \rightarrow k^{+} \Sigma^{-}\right)=0 \\
& \frac{d}{d \Omega} \sigma\left(\pi^{-} p \rightarrow k^{+} \Sigma^{-}\right)=0 .
\end{aligned}
$$

On the basis of equation (21), and of the charge independence, one derives further the following additional relations: 


$$
\begin{aligned}
\frac{d}{d \Omega} \sigma\left(\pi^{+} p \rightarrow k^{+} \Sigma^{+}\right) & =2 \frac{d}{d \Omega} \sigma\left(\pi^{-} p \rightarrow k^{0} \Sigma^{0}\right), \\
\frac{d}{d \Omega} \sigma\left(k^{-} p \rightarrow \pi^{-} \Sigma^{+}\right) & =2 \frac{d}{d \Omega} \sigma\left(k^{-} n \rightarrow \pi^{-} \Sigma^{0}\right)= \\
& =4 \frac{d}{d \Omega} \sigma\left(k^{-} p \rightarrow \pi^{0} \Sigma^{0}\right)
\end{aligned}
$$

which may be tested in the future.

As we emphasized often, in all these derivations, we did not really assume the validity of exact SU(3) symmetry, provided that the energy is high enough that one could neglect the mass of the projectile mesons. So in a sense, these relations so far derived do not really test the SU(3) symmetry. However, if we assume now the exact validity of the SU(3) symmetry in addition to our ansatz equation (4), then one can derive a few more relations among various scattering amplitudes such as

$$
\begin{aligned}
T\left(\pi^{+} p \rightarrow k^{+} \Sigma^{+}\right)= & T\left(k^{+} p \rightarrow k^{+} p\right)-T\left(\pi^{+} p \rightarrow \pi^{+} p\right) \\
T\left(k^{-} p \rightarrow \pi^{-} \Sigma^{+}\right)= & T\left(k^{-} p \rightarrow k^{-} p\right)-T\left(\pi^{-} p \rightarrow \pi^{-} p\right) \\
\sqrt{6} T\left(\pi^{-} p \rightarrow k^{0} \Lambda\right)= & T\left(k^{+} p \rightarrow k^{+} p\right)-T\left(\pi^{+} p \rightarrow \pi^{+} p\right)+ \\
& +2\left[T\left(k^{-} n \rightarrow k^{-} n\right)-T\left(k^{-} p \rightarrow k^{-} p\right)\right] \\
2 \sqrt{3} T\left(k^{-} p \rightarrow \pi^{0} \Lambda\right) & =T\left(\pi^{-} p \rightarrow \pi^{-} p\right)-T\left(k^{-} p \rightarrow k^{-} p\right)+ \\
& +2\left[T\left(k^{-} n \rightarrow k^{-} n\right)-T\left(\pi^{+} p \rightarrow \pi^{+} p\right)\right]
\end{aligned}
$$

Again, these are independent of the special assumption which led to equation (17). Unfortunately these cannot be directly tested experimentally. However, one may get the familiar triangular relations:

$$
\begin{aligned}
& {\left[\frac{d}{d \Omega} \sigma\left(\pi^{+} p \rightarrow \pi^{+} p\right]^{1 / 2} \leqslant\left[\frac{d}{d \Omega} \sigma\left(k^{+} p \rightarrow k^{+} p\right]^{1 / 2}+\left[\frac{d}{d \Omega} \sigma\left(\pi^{+} p \rightarrow k^{+} \Sigma^{+}\right]^{1 / 2} .\right.\right.\right.} \\
& {\left[\frac{d}{d \Omega} \sigma\left(\pi^{-} p \rightarrow \pi^{-} p\right]^{1 / 2} \leqslant\left[\frac{d}{d \Omega} \sigma\left(k^{-} p \rightarrow k^{-} p\right]^{1 / 2}+\left[\frac{d}{d \Omega} \sigma\left(k^{-} p \rightarrow \pi^{-} \Sigma^{+}\right]^{1 / 2}\right.\right.\right.}
\end{aligned}
$$

These may be used as a test of the validity of the SU(3) symmetry at the high energy limit. 


\section{Connection with Regge Poles}

In the previous section, we noted how naturally our theory leads to the investigation of the $t$-channel in the high energy behavior and also we can understand why the Barger-Rubin relation and others are well satisfied experimentally. Here, we shall show in some details that our approach seems to have a close relationship with the theory based upon the Regge pole. Indeed, we can make an almost one to one correspondence between our results and those of the Regge pole, as we have partially seen in the previous section.

First as a preliminary, one notes from equation (4) that

$$
\begin{aligned}
& Q_{0}(-x)=-Q_{0}(x) \\
& Q_{b}^{a}(-x)=+Q_{b}^{a}(x) \\
& \bar{Q}_{b}^{a}(-x)=-\bar{Q}_{b}^{a}(x)
\end{aligned}
$$

However, we must be cautioned that equation (25) does not imply that $Q_{0}(x), \bar{Q}_{b}^{a}(x)$ have the natural parity -1 while $Q_{b}^{a}(x)$ has the parity +1 since these quantities are not local operators. Also, because of the self-conjugate property of the meson field

$$
\left(j_{b}^{a}(x)\right)^{+}=j_{a}^{b}(x)
$$

one shall have the following:

$$
\begin{aligned}
& Q_{0}^{+}(x)=Q_{0}(-x) \\
& {\left[Q_{b}^{a}(x)\right]^{+}=Q_{a}^{b}(-x)} \\
& {\left[\bar{Q}_{b}^{a}(x)\right]^{+}=\bar{Q}_{a}^{b}(-x) .}
\end{aligned}
$$

Now, for simplicity, let us consider the case where the states $|\alpha\rangle$ and $|\beta\rangle$ in equation (1) are spinless one particle states with four momenta $p$ and $p^{\prime}$, respectively. Then, because of the Lorentz covariance, one may set

$$
\left.<p^{\prime}\left|Q_{A}(x)\right| p\right\rangle=\left(\frac{1}{4 p_{0} p_{0}} \cdot\right)^{1 / 2} F_{A}\left(p^{\prime} x, p x, x^{2}\right)
$$

where $Q_{A}(x)$ represents one of $Q_{0}(x), Q_{b}^{a}(x)$ and $\bar{Q}_{b}^{a}(x)$, and where $F_{A}$ are the corresponding scalar functions of $p^{\prime} x, p x$ and $x^{2}$ as well as the square of the momentum transfer. It is now not difficult to show that time-reversal invariance of the theory assures us that we should have

$$
\begin{aligned}
& <p^{\prime}\left|Q_{0}(x)\right| p>=\text { pure imaginary } \\
& <p^{\prime}\left|Q_{b}^{a}(x)\right| p>=\text { pure real }
\end{aligned}
$$




$$
<p^{\prime}\left|\bar{Q}_{b}^{a}(x)\right| p>=\text { pure imaginary }
$$

We remark that this can be generalized to the case when states $|\alpha\rangle$ and $|\beta\rangle$ possess the spin $1 / 2$. In that case, we decompose

$$
\left\langle p^{\prime}\left|Q_{A}(x)\right| p\right\rangle=\left(\frac{1}{p_{0} p_{0}}\right)^{1 / 2} \bar{u}\left(p^{\prime}\right)\left\{F_{A}+\left[i \gamma x, \gamma\left(p^{\prime}-p\right)\right] G_{A}\right\} u(p)
$$

instead of equation (27), where $F_{A}$ and $G_{A}$ are again scalar functions of $p^{\prime} x, p x$ and $x^{2}$. Similarly, the time-reversal invariance demands that $F_{0}, G_{0}, \bar{F}_{b}^{a}$ and $\bar{G}_{b}^{a}$ are all purely imaginary numbers while $F_{b}^{a}$ and $G_{b}^{a}$ are purely real.

Now, after these preliminaries, we shall show how the diffraction shrinkage may be obtained in our theory. We noted already that the high energy phenomena may be determined by the property of the retarded commutator on the light cone. Hence, because of causality or from the inspection of the Dyson's representation [8] we conjecture that $F_{A}$ may be proportional to $\delta\left(x^{2}\right)$ $\delta^{\prime}\left(x^{2}\right), \theta\left(-x^{2}\right)$, or in any linear combination of these on the light cone. of course, these considerations do not tell much about the dependence of $F_{A}$ on $p^{\prime} x$ and $p x$. As a simple choice, we conjecture that it may have the following forms:

$$
F_{0}(x)=-i \gamma(t) \cdot \varepsilon\left(x_{0}\right) \cdot \delta^{\prime}\left(x^{2}\right)\left|\left(\frac{p^{\prime}+p}{2} \cdot x\right)\right|^{-\alpha(t)}
$$

where $t$ is the square of the momentum transfer, i.e.,

$$
t=-\left(p^{\prime}-p\right)^{2}=-\left(k^{\prime}-k\right)^{2}
$$

and $\alpha(t)$ and $\gamma(t)$ are some functions of $t$. The factor $\varepsilon\left(x_{0}\right)$ has been introduced in conformity with the condition equation (25). Similarly for $F_{b}^{a}(x)$ we replace $i \varepsilon\left(x_{0}\right)$ by unity in equation (30), with new sets of $\alpha(t)$ and $\gamma(t)$. As for $\bar{F}_{b}^{a}(x)$ we may use equation (30) with another set of $\alpha(t)$ and $\gamma(t)$. Then the time-reversal requirement equation (28) implies that $\alpha(t)$ and $\gamma(t)$ must be real functions of $t$.

Now, let us set

$$
T_{0}(i \rightarrow f)=\left(16 k_{0} k_{0} p_{0} p_{0}^{\prime}\right)^{-1 / 2} \cdot M_{0}(s, t)
$$

and

$$
M_{0}(s, t)=i \int d^{4} x \exp \left[i \cdot \frac{k+k^{\prime}}{2} x\right]\left\langle p^{\prime}\left|\theta\left(-x_{0}\right) Q_{0}(x)\right| p\right\rangle
$$

where $T_{0}$ is the part of the scattering amplitude from the $Q_{0}(x)$ term. Similarly, one defines $T_{b}^{a}$ and $\bar{T}_{b}^{a}$. Also, in equations (32) $s$ is of course the energy variable given by

$$
s=-(p+k)^{2}=-\left(p^{\prime}+k^{\prime}\right)^{2}
$$


It is simple now to compute $M_{0}(s, t)$ by inserting the ansatz equation (30) into the R.H.S. of equation (32b) to obtain

$$
\begin{gathered}
M_{0}(s, t)=-\frac{\pi}{2}\left(m^{2}-\frac{t}{4}\right)^{-1 / 2 \alpha(t)} \cdot \gamma(t) \cdot \Gamma(-\alpha) \cdot \exp \left[-\frac{\pi}{2} i \alpha(t)\right] \times \\
\times\left[(\omega-k)^{\alpha(t)}+(\omega+k)^{\alpha(t)}\right]
\end{gathered}
$$

where $\omega$ and $k$ are given by

$$
\begin{aligned}
& \omega=\frac{1}{2}\left(m^{2}-\frac{t}{4}\right)^{-1 / 2} \cdot\left[s-m^{2}-\mu^{2}+\frac{t}{2}\right] \\
& k=\left[\omega^{2}-\mu^{2}+\frac{t}{4}\right]^{k / 2}
\end{aligned}
$$

and where $m$ and $\mu$ are masses of the incoming meson and of the target, respectively. Also, in the derivation of these equations, one assumed for simplicity that we are dealing with the elastic scattering or that we can neglect the mass differences among incoming and outgoing particles. Equation (34) can be derived on the basis of the following formula [9] for the Fourier transform of generalized functions:

$$
\int_{0}^{\infty} d x|x|^{\lambda} \cdot \exp (i \sigma x)=i \Gamma(\lambda+1) \cdot e^{\frac{\pi}{2} \lambda i} \cdot\left(\sigma+i_{0}\right)^{-(\lambda+1)} .
$$

When we let $s \rightarrow \infty$ in equation (34), one immediately finds then

$$
M_{0}(s, t) \approx-\frac{\pi}{2}\left(m^{2}-\frac{t}{4}\right)^{-\alpha(t)} \gamma(t) \Gamma(-\alpha) \exp \left[-\frac{\pi}{2} i \alpha(t)\right] \cdot s^{\alpha(t)}
$$

for $\alpha(t)>0$, and

$$
M_{0}(s, t) \approx-\frac{\pi}{2}\left(\mu^{2}-\frac{t}{4}\right)^{-\alpha(t)} \cdot \gamma(t) \Gamma(-\alpha) \exp \left[-2_{2} \iota \alpha(t)\right] s^{-\alpha(t)}
$$

for $\alpha(t)<0$. At any rate, we see that $M_{0}(s, t)$ is proportional to $s|\alpha(t)|$ for $s \rightarrow \infty$, which exhibits the Regge behavior. On this connection, one may remark that the choice of equation (30) is not the only one to lead to the Regge behavior. Indeed, we shall obtain a similar behavior if we have

$$
F_{0}(x)=-i \gamma(t) E\left(x_{0}\right) \delta\left(x^{2}\right)\left|\left(\frac{p+p^{\prime}}{2}\right) x\right|^{\alpha(t)}
$$

or

$$
F_{0}(x)=-i \gamma(t) \varepsilon\left(x_{0}\right) \delta\left(x^{2}\right) \cdot P_{\alpha}\left(\left\|\frac{p+p^{\prime}}{2} x\right\|\right)
$$

instead of equation (30). Anyhow, the important point is that in fact we can reproduce a Regge behavior within the framework of our theory, although admittedly only by heuristic assumptions.

Now, we could interpret our $\alpha(t)$ and $\gamma(t)$ in our approach to be the slope and the residue of the Regge trajectory, respectively. Then it is very gratifying that both $\alpha(t)$ and $\gamma(t)$ must 
be real functions of $t$ as we have shown by means of the time-reversal invariance. The reality of these functions for $t \leqslant 0$ is already known in the ordinary Regge pole model. We may repeat exactly the same calculation for scattering amplitudes involving $Q_{b}^{a}(x)$ and $\bar{Q}_{b}^{a}(x)$. Then, because of the time reversal requirement equation (28), we must now have a phase difference of $90^{\circ}$ between matrix elements of $Q_{b}^{a}(x)$ and $\bar{Q}_{b}^{a}(x)$. This is the reason why we must replace $i \varepsilon\left(x_{0}\right)$ in equa tion (30) by unity for $Q_{b}^{a}(x)$ but use the same formula for $\bar{Q}_{b}^{a}(x)$. Then, assuming the same $x^{2}$ and $\left(p+p^{\prime}\right) \cdot x$ dependencies for these functions as in equation (30) with different sets of $\alpha(t)$ and $\gamma(t)$, one again obtains a formula similar to equations (37). Thus we find that the scattering amplitude $T_{b}^{a}$ and $\bar{T}_{b}^{a}$ must have the phase difference of $90^{\circ}$, apart from a kinematicai factor $\exp \cdot\left[\frac{\pi}{2} i \alpha(t)\right]$. Actually, this is exactly what we expect from the Regge pole model since both $1^{-}$and $2^{+}$trajectories have different kinematical dependences of $[1 \mp \exp (-i \pi \alpha)]$ because of the difference of signature of the two trajectories. Also, experimentally, the Regge trajectory $\alpha(t)$ appears to be almost the same for both $1^{-}$and $2^{+}$nonets if we compare those with the same charge and strangeness. Then, one concludes that contributions to the scattering amplitudes frop $Q_{b}^{a}(x)$ and $\bar{Q}_{b}^{a}(x)$ must have exactly the $90^{\circ}$ phase difference. This fact has been already utilized heavily in the previous section to derive equations (14) and (17). Of course, these facts are already known in the Regge pole model [7], but we gave a different interpretation for the signature factor on the basis of the time-reversal invariance.

Thus, we can reproduce essentially all results of the Regge pole model at high energy without introducing the concept of complex angular momentum. Also, it is clear now that $Q_{0}(x)$ and $Q_{b}^{a}(x), \bar{Q}_{b}^{a}(x)$ can be regarded to correspond to the Pomeranchukon, $1^{-}$vector nonet and $2^{+}$tensor nonet trajectories, respectively. It is quite interesting that our way of introducing the nonet of vector mesons is exactly the same as what has been postulated earlier [10] phenomenologically in connection with the $\omega-\phi$ mixing. It is also known to be experimentally compatible [11] with the high-energy meson-baryon scattering data. As for the tensor nonet, the same remark holds valid also [12], although at first glance we seem to be introducing the traceless part $\bar{Q}_{f}(x)$ in equation (5c). However, the presence of this term does not affect anything except possibly for the case of $\eta+\alpha \rightarrow \eta+\beta$ scattering, which we are not interested in. Hence, again, our procedure is practically the same as the ordinary treatment of the tensor nonet, which is known to be consistent with experiment $[7,11]$.

So far, we have completely neglected the consideration of the $R_{b d}^{a c}(x)$ term in equation (3). We already remarked a few times that it does not contribute at all for the total scattering cross sections. Here we shall present an argument that it can be neglected also for elastic scattering problems. Let us set

$$
T_{R}=i\left(4 k_{0} k_{0}^{\prime}\right)^{-1 / 2} \cdot \int d^{4} x \exp \left[i \frac{k+k^{\prime}}{2} x\right] \cdot<p^{\prime}\left|R_{b d}^{a c}(x)\right| p>\text {. }
$$

Integrating by parts with respect to $x_{0}$ and using the translational invariance of the theory, one can rewrite it as follows:

$$
T_{R}=T_{R}^{(1)}+T_{R}^{(2)} \text {, }
$$




$$
\begin{aligned}
& T_{R}^{(1)}=-i\left(4 k_{0} k_{0}\right)^{-1 / 2} \int d^{4} x \exp \left[i \frac{k+k^{\prime}}{2} x\right] \times \\
& \mathrm{x}\left\{\left\langle p^{\prime}\left|\delta\left(x_{0}\right)\left[j_{b}^{a}\left(\frac{x}{2}\right), \frac{\partial}{\partial x_{0}} \varphi_{d}^{c}\left(-\frac{x}{2}\right)\right]\right| p\right\rangle+\left\langle p^{\prime}\left|\delta\left(x_{0}\right)\left[\frac{\partial}{\partial x_{0}} \Phi_{b}^{a}\left(\frac{x}{2}\right), j_{d}^{c}\left(-\frac{x}{2}\right)\right]\right| p>\right\},\right. \\
& T_{R}^{(2)}=\frac{1}{2} \cdot\left(4 k_{0} k_{0}^{\prime}\right)^{-1 / 2} \cdot \int d^{4} x \exp \left[i \frac{k+k^{\prime}}{2} x\right] \times \\
& x\left\{k_{0}{ }^{\prime}<p^{\prime}\left|\delta\left(x_{0}\right)\left[\varphi_{b}^{a}\left(\frac{x}{2}\right), j_{d}^{c}\left(-\frac{x}{2}\right)\right]\right| p>+\right. \\
& \left.+k_{0}<p^{\prime}\left|\delta\left(x_{0}\right)\left[j_{b}^{a}\left(\frac{x}{2}\right), \Phi_{d}^{c}\left(-\frac{x}{2}\right)\right]\right| p>\right\}
\end{aligned}
$$

Now, we expect that equal-time commutators inside equations (40b) and (40c) would become proportional to four-dimensional delta functions $\delta^{(4)}(x)$ because of causality and covariance. Then, at the high energy limit, $T_{R}(1)$ will behave as $s^{-1}$ for $s \rightarrow \infty$ and hence can be neglected in comparison with contributions from the Regge-type terms which give rise to $s^{\alpha-1}(\alpha>0)$. However, the second term $T_{R}(2)$ in equation (40c) might give a constant contribution in the $s \rightarrow \infty 1$ imit unless it vanishes identically. If so, it could not be neglected at all. Here, we shall show that on the basis of the ordinary canonical commutation relation we should have $T_{R}(2) \equiv 0$. To prove it, we modify the commutator inside equation (40c) as follows:

$$
\begin{aligned}
& <p^{\prime}\left|\delta\left(x_{0}\right)\left[\Phi_{b}^{a}\left(\frac{x}{2}\right), j_{d}^{c}\left(-\frac{x}{2}\right)\right]\right| p>= \\
& =\left\langle p^{\prime}\left|\delta\left(x_{0}\right)\left[\Phi_{b}^{a}\left(\frac{x}{2}\right),\left(\mu^{2}-\frac{1}{4} \Delta_{x}\right) \Phi_{d}^{c}\left(-\frac{\dot{x}}{2}\right)\right]\right| p>+\right. \\
& +4<p^{\prime}\left|\delta\left(x_{0}\right)\left[\frac{\partial}{\partial x_{0}} \Phi_{b}^{a}\left(\frac{x}{2}\right), \frac{\partial}{\partial x_{0}} \Phi_{d}^{c}\left(-\frac{x}{2}\right)\right]\right| p>- \\
& -2 i\left(p_{0}^{\prime}-p_{0}\right)<p^{\prime}\left|\delta\left(x_{0}\right)\left[\Phi_{b}^{a}\left(\frac{x}{2}\right), \frac{\partial}{\partial x_{0}} \Phi_{d}^{c}\left(-\frac{x}{2}\right)\right]\right| p>
\end{aligned}
$$

where we used equation (2) together with the translational invariance of the theory. However, the canonical commutation relations at equal time demands that all these terms must vanish. For example, the last term will becume

$$
-\left(p_{0}^{\prime}-p_{0}\right) \cdot z_{3}-1 \cdot \delta^{(4)}(x)<p^{\prime} \mid p>\cdot\left[\delta_{d}^{a} \delta_{b}^{c}-\frac{1}{3} \delta_{b}^{a} \delta_{d}^{c}\right]=0
$$


We can prove the same for the second term for the R.H.S. of equation (40c) and hence we conclude $T_{R}(2) \equiv 0$. Therefore, one can justify neglecting the contribution from the $R_{b d}^{a c}(x)$ term in all situations.

Returning to our original problem, one may note that we could incorporate a part of a recent speculation of Cabbibo, Horwitz and Ne' eman [13] naturally in our model. We have remarked already that the $\bar{Q}_{b}^{a}(x)$ term is essentially related to the $2^{+}$tensor nonet trajectory. Besides, in our theory, it is a scalar function of $x$ as is clear from the original definition equation (4). Hence, it is natural to conjecture that it could be proportional to the scalar density operator $u_{b}^{a}(0)$ of Gell-Mann [5] in the quark model, apart from some kinematical factors like $\varepsilon\left(x_{0}\right) \delta^{\prime}\left(x^{2}\right)$ as in equation (30). Then, the Regge residue function $\gamma(t)$ is essentially proportional to the matrix element of $u_{b}^{a}(0)$. Further, one notes that in the quark model, it is given by

$$
u_{b}^{a}(x)=\bar{q}_{a}(x) \quad q_{b}(x)-\frac{1}{3} \delta_{b}^{a} \quad \bar{q}_{c}(x) q_{c}(x)
$$

where $q_{a}(x),(a=1,2,3)$ is the quark field. Thus, one may moreover assume that $u_{3}^{3}(x)$ is the mass-breaking medium strong interaction [3] of the SU(3) theory. Then one can immediately compute the $\bar{f} / \bar{d}$ ratio of the coupling of $\bar{Q}_{b}^{a}(x)$ with the baryon octet in the case of the forward scattering by the formula:

$$
\bar{f} / \bar{d}=-\frac{2}{3} \cdot \frac{m(\equiv)-m(N)}{m(\Sigma)-m(\Lambda)} \approx-3.3
$$

On the basis of equation (41), one can derive the following sum rule, provided that we assume now the validity of the SU(3) symmetry:

$$
\begin{aligned}
& {[m(\equiv)-m(\Sigma)] \cdot\left[\sigma_{t}\left(\pi^{+} p\right)+\sigma_{t}\left(\pi^{-} p\right)\right]+[m(\Sigma)-m(N)] \cdot\left[\sigma_{t}\left(k^{+} n\right)+\sigma_{t}\left(k^{-} n\right)\right]=} \\
& \quad=[m(\equiv)-m(N)] \cdot\left[\sigma_{t}\left(k^{+} p\right)+\sigma_{t}\left(k^{-} p\right)\right]
\end{aligned}
$$

where we used the mass formula $3 m(\Lambda)+m(\Sigma)=2[m(\equiv)+m(N)]$ in order to simplify the result and also we have set all $\alpha(0)$ for the tensor trajectories to be the same in conformity with the SU(3) symmetry. We note that this relation is satisfied experimentally with 10 per cent error for the mesons laboratory momentum of more than $10 \mathrm{Bev} / \mathrm{C}$. Besides, one notes that $\bar{f} / \bar{d} \approx-3.3$ is quite near [11] to the experimental $f / d=-2.0 \pm 0.4$ for the $1^{-}$vector nonet coupling with the baryon. This may be taken for another confirmation of the phenomenological assumption that the $1^{-}$and $2^{+}$nonets couple universally [7] with baryons except for the difference of the signature factors.

Similarly, we can compute the ratio of the residues of the $R$-trajectories for $k$ an and nucleon by

$$
\gamma_{N}^{(R)} \quad \gamma_{k}^{(R)}=\frac{4}{3} \cdot \frac{m(\equiv)-m(\Sigma)}{m(\eta)-m(\pi)} \cdot \frac{m(N)}{m(k)} \approx 0.75
$$


where we used the linear mass formula for both mesons and baryons. When we use the quadratic mass formula for mesons, then equation (43a) must be replaced by

$$
\gamma_{N}^{(R)} \quad \gamma_{k}^{(R)}=\frac{4}{3} \cdot \frac{m(\equiv)-m(\Sigma)}{m^{2}(\eta)-m^{2}(\pi)} \cdot ل_{2}\left[m^{2}(\eta)+m^{2}(\pi)\right]^{1 / 2} \cdot \frac{m(N)}{m(k)} \approx 0.81 .
$$

These numbers may be compared to the corresponding one [11] for the $1^{-}$nonet of

$$
0.5<\frac{\gamma N}{\gamma M}<1.5
$$

and our values are compatible with the expected universality assumption.

Similarly, it is possible to assume that $Q_{b}^{a}(x)$ mas be proportional to the vector octet current $\left[v_{\mu}(x)\right]_{b}^{a}$ in the quark model. Then, for non-spin flip amplitude in the forward scattering problem, one would expect to have $\left\langle n\left|Q_{1}^{1}\right|_{n}>\alpha G_{E}^{(n)}(0)=0\right.$ where $G_{E}^{n}(t)$ is the electric form factor of the neutron. However, this gives rise to $d / f=0$ and also to

$$
\sigma_{t}\left(\pi^{-} p\right)-\sigma_{t}\left(\pi^{+} p\right)=\sigma_{t}\left(k^{-} p\right)-\sigma_{t}\left(k^{+} p\right)
$$

which is nothing but one of the Johnson-Treiman sum rules and is known [2] to disagree with the experiment by nearly a factor of 2 . Hence, this assumption may not be so good. Besides, we have trouble in how to equate a scalar function $Q_{b}^{a}(x)$ with a vector $\left[v_{\mu}(x)\right]_{b}^{a}$ in this assumption.

Last, one may mention that the same technique may be used to determine the high momentum transfer limit of the electromagnetic form factors of the nucleon. This will be treated elsewhere.

\section{Acknowledgements}

This paper was initiated when the author was staying at the International Center for Theoretical Physics, Trieste, Italy. He would like to express his gratitude to Professors A. Salam and P. Budini, and I. A. E. A. for hospitality. He is also thankful for a John Simon Guggenheim Fellowship.

\section{References}

1. S. OKUBO, Nuovo Cim. 44, 276 (1966).

2. V. BARGER and M.H. RUBIN, Phys. Rev. 140, B 1365 (1965).

3. S. OKUBO, Prog. theor. Phys., Kyoto 27, 949 (1962).

4. See for instances; Introduction to Fourier Analysis and Generalized Functions, by M.J. LIGHTHILL, lecture note, University of Manchester. (See also Ref. 9). 
5. M. GELl-MANN, Phys. Rev. 125, 1067 (1962); and Physics 1, 63 (1964).

6. B.M. UdGaONKAR and M. GELL-MANN, Phys. Rev. Letters 8, 147 (1962).

7. A. AHMADZADEH and C.H. CHAN, University of California preprint (1966); A. AHMADZADEH, Phys. Rev. Letters, 16, 952 (1966).

8. F.J. DYSON, Phys. Rev. 110, 1460 (1958).

9. I.M. GEL' FAND and G.E. SHILOV, Generalized Functions, Vol. 1. Academic Press, New York (1964).

10. S. OKUBO, Phys. Letters 5, 165 (1963).

11. V. BARGER and M. OLSSON, Phys. Rev. Letters 15, 930 (1965).

12. S.L. GLASHOW and R.H. SOCOLOW, Phys. Rev. Letters 15, 329 (1965).

13. N. CABIBBO, L. HORWITZ and Y. NE' EMAN, Phys. Letters 22, 336 (1966).

\section{Note added in proof}

Some of the sum rules derived in this paper may also be obtained by intermediate coupling theory. [See J. KURIYAN and E.C.G. SUDARSHAN, Phys. Rev. Letters 16, 825 (1966).]

The author would like to express his gratitude to Prof. Sudarshan for pointing out this fact. 